\title{
Using Location Lifelogs to Make Meaning of Food and Physical Activity Behaviors
}

\author{
Adrienne H. Andrew, Kevin Eustice, Andy Hickl \\ ARO, Inc. \\ Seattle, WA \\ \{aha,k, a\}@aro.com
}

\begin{abstract}
In this paper, we take the position that location and information derived from location adds value to health and wellness logs. Traditional health and wellness logs such as food and physical activity diaries do not include location information in a meaningful way. As the ability to track and make sense of location is improving, it will be possible to associate high-quality data regarding the user location and location-derived activities to health and wellness logs. This can significantly improve the ability of these logs to expose meaning to the user, specifically around food and physical activity behaviors. We address four dimensions of location related information: location in terms of places and travels; information derived from location such as roles and transitions; routines identified from places and place transitions; and finally, identifying cohorts and aggregating over routines.
\end{abstract}

Keywords-lifelog; location; food diary; physical activity sensing; routine identification; behavioral tendencies; cohort identification;

\section{INTRODUCTION}

Lifelogging is loosely defined as capturing and storing detailed information about one's life. It is different than simple journaling, which consists of recording a synthesized version of one's experiences. A key feature of lifelogging is capturing as much detail as possible, over a long period of time. Buckminster Fuller began his "lifelog" via scrapbook in the 1920 s, capturing his life by documenting what he was doing every 15 minutes. Advances in technology have supplanted this manual process. In the 1990 s, Steve Mann began wearing a video camera to capture a continuous video of his life experiences. The development of the SenseCam has enabled lifelogging to transform from a manually intensive process to one that can passively capture huge amounts of data.

The Quantified Self (QS) community presents a slightly different approach to lifelogging. QS enthusiasts typically begin with the goal of capturing and understanding one (or a few) very specific, well-defined aspects of their life. The intent is to better understand or improve something about of interest. Benjamin Franklin is frequently identified as an early QS practitioner, taking time each day to monitor whether he practiced the virtues he valued. He did this by filling out a chart that he designed to help with his data collection and analysis. Self-monitoring is an important tool for helping people to capture, reflect on and modify their behavior, but a manual data collection and analysis process makes it difficult. Modern technology in the form of spreadsheets, websites and mobile phone apps have made it easier to capture, analyze and share data.

The different goals behind lifelogging and self-monitoring has are reflected in different research focuses. The goal of the lifelog is usually to collect as much data about an individual's life as possible, and has taken the approach of trying to capture an individual's experience of life. This resulted in an emphasis on capturing images, video, sound, location, and email. Email helped to capture social relationships, communication patterns, general life busy-ness, and concepts or problems the individual was spending time on. Lifelogging research includes how to capture these large amounts of data, store them efficiently, and access them appropriately.

On the other hand, individuals interested in quantified self $\log$ very specific data, usually with the goal of answering a specific question, support a desired behavior change, or simply to "know thyself". Some of the things that quantified selfers monitor are very difficult to capture via technology and rely on the individual to manually monitor and/or self-report (such as mood or food eaten). Research concerns around selfmonitoring place more emphasis on inspiring motivation, sustaining engagement, and influencing changes in behavior over time.

Western countries have an increasing number of lifestyle diseases such as diabetes and obesity. One approach to address the epidemic is to provide tools to help individuals understand the impact of their behaviors. The Journal of Preventive Medicine recently focused on the promise of visual lifelogs for improving behaviors associated with lifestyle diseases [1]. In this position paper, we argue that location lifelogs plus health and wellness data improves the knowledge that users can get from that data. Behavior change is about a series of small changes, and making sure those changes trend in the desirable way. Location plus health and wellness data could be not just interesting, but help people to change their behavior, recognize when their behavior is changing, decide whether that is a good or bad thing, and then react. Specifically, one complaint against most self-monitoring tools is that while they are good at identifying the indicators of one's behavior, they are not very good at providing actionable intelligence. For example, users often report "I know that I only take $5 \mathrm{k}$ steps per day, and advice to walk more isn't terribly helpful".

The rest of this position paper is structured into three sections. First, we describe some background technologies and 
capabilities around capturing and making sense of location. Then we briefly describe an example data collection platform. Finally, we detail the benefit that different location analyses can have on the use of health and wellness data.

\section{BACKGROUND}

Our discussion builds on three bodies of research: automatic geolocation, persuasive technology for behavior change, and self-monitoring technologies. This background is far from complete, but provides evidence that the capabilities we describe are plausible in the near-term.

\section{A. Automatic Geolocation}

Modern mobile phones can passively collect location lifelogs using a combination of Wi-Fi access points [2] and GPS [3] to identify where people are, when they are there, and where they will go next. Ashbrook and Starner [3], Hightower et al [4] and Kang et al [5] show how meaningful places can be identified from location lots and how to extract a semantically meaningful place from location data.

One limitation to all of these capabilities has been putting them together into a single device that has the resources to do this data collection and sensemaking in a continuous fashion. However, the improved capabilities of mobile devices, availability of networks, and access to cloud resources for processing and storage has made it possible to combine all of these capabilities into a location-based lifelogging platform. This is evidenced in mobile apps such as Google's Latitude.

Machine learning can be used to extract recurrent patterns of locations from location streams [6], [7]. Using LDA, Ferrari et al. were able to find different patterns for different days of the week for each person (e.g. Pub-Home-Pub on Saturday).

\section{B. Persuasive Technology to Support Behavior Change}

Persuasive technology is technology designed to change behavior or attitudes. Technology can play three different roles: tools, or as a means to help with a task; media, or as a means to provide content; or social actor, or like a living entity [8]. Technology can change attitudes or behaviors by engaging these three different roles to make desired outcomes easier to achieve. Fogg identifies seven types of tools that support this [8]. Reduction supports the task of change by simplifying something that is challenging or complex. Tunneling guides the individual through the process of change. Tailoring provides customized information for an individual, which makes it easier for the individual to understand, relate to, and take action on. Suggestion uses context to intervene at the moment of maximum relevance. Self-monitoring makes it easy or enjoyable to monitor and reflect on their attitudes or behaviors. Surveillance allows an individual to be monitored, supporting behavior change. Conditioning uses principles of operant conditioning to change behavior by reinforcing the use of new behaviors. Finally, while we are discussing these strategies as applied to the design of computational technologies, they also apply to non-technological implementations. For example, selfmonitoring can be done with pencil and paper.

\section{Self-Monitoring of Food Intake and Physical Activity}

Self-monitoring is an important tool to support people in changing their eating and physical activity behaviors to support lifestyle disease prevention. Traditionally, self-monitoring of food intake is a manual process. Mobile phones and websites now provide tools that make it easier to capture detailed food intake information. However, all tools require diligence on behalf of the user. Typically, the user must find the food in a database, estimate how much was eaten, and perhaps specify some relevant metadata such as time eaten or which meal. Some food diaries allow the user to take a photo, either as the record or as a "bookmark" to provide context to enter the details later. Increasingly, photography is being used to improve logging of food intake. Zhu et al. [9] (and others) have used smartphones to take photos of food before and after eating in order to use computer vision techniques to identify how much of what food was eaten, and lookup the amount of nutrients consumed. The use of visual lifelogging tools (such as the SenseCam) that automatically capture food photographs improves the identification of how much of what food was eaten [10].

While accurate self-monitoring of food intake is still a fairly manual process (although automation is improving), many tools accurately capture physical activity. Tools to support tracking physical activity data depend on a range of input effort from the user. MyFitnessPal is an example of a mostly manual physical activity record that requires the user to enter all the data manually. RunKeeper, Endomondo and MapMyFitness are examples of mobile phone apps that require the user to specify when they are starting a specific activity, but then automatically capture duration, speed, location traces, and other details about the exercise bout. FitBit, BodyMedia FIT, and Jawbone UP are devices that a user wears all day. The devices passively capture movement throughout the day, and uses that information to identify activity bouts, intensity of activity, and incidental activity, then calculate activity measurements. Calculated measures vary from device to device.

\section{ENVISIONED TECHNOLOGY}

Today, existing components can be combined to create a location-based lifelogging platform for food and activity behaviors. In this section, we describe a smartphone-based system that supports the collection of location, food intake and physical activity data. An example instantiation of this system could be a combination of three tools: a passive location collection tool (such as Google Latitude); a food diary (such as LoseIt); and a physical activity sensor (such as the BodyMedia FIT). The location collection tool provides GPS traces from which places can be reverse geocoded. The food diary supplies when which foods have been eaten, supplying calories consumed. The physical activity sensor provides a reliable calculation of energy expenditure over a period of time. While the food diary relies on self-report (manual entry by the user), the other two components are primarily passive collection.

\section{The Value of Location}

We propose that there are four ways location can provide value to food and physical activity diaries. In this section, we describe these four ways, which we refer to as "levels" and show how they improve a lifelog. We also provide examples of how these levels could benefit the user. 


\section{A. Stay and Travel Segmentation}

The first level of meaning we can make from a location trace is to segment stays and travels. A stay is a period of time when a users remains in one location, while a travel is the time and path between two stays. Using reverse geocoding, we can identify something about the places where a stay segment occurs, such as an address or even business name. As part of this, we may even be able to identify what kind of business it is from available lookup services.

There are three primary technical challenges for parsing location traces into stays and travels. First is the availability of location detection. GPS is a good location provider, but is often unavailable indoors and fails predictably when the GPS receiver does not have line of sight with enough satellites. Assisted GPS (aGPS) and Wi-Fi localization improve location tracking ability, including indoors. The second challenge is determining the size of a place when distinguishing between a stay and a travel. For example, consider a person walking around a small apartment as opposed to walking from gate to gate at a large airport. A third challenge to parsing location into stays and travels is choosing the minimum duration for identifying a stay. Contrast two scenarios: (A) A user is driving in heavy traffic and is stuck at a red light for a few minutes; (B) A user is driving, stops to get gas, and returns to driving a couple of minutes later. The amount of time spent in each location is similar, but the user probably considers the time at the red light as part of the travel, while the time at the gas station is a distinct stay.

Identifying location with physical activity data (such as heart rate, number of steps taken, number of calories burned) can help to identify incidental rather than intentional physical activity. It can also provide information about calories burned or consumed in a particular location. This enables the system to answer questions the user might have such as:

- How much time do I spend in one place versus between places?

- How many different places do I go in a day?

- Where am I most stressed?

- Where do I burn the most calories?

- How does the amount of time I spend in one location correlate with my calorie burn?

- How many restaurants do I go to?

The range of questions we can answer by simply combining stay and travel information with food and activity data is limited without more detailed information. However, it is likely that this level of information is enough to provide contextual reminders to support the data collection that requires manual input, such as the food diaries.

\section{B. Roles and Transitions Identification}

The next level of meaning is to interpret the set of stays collected for a user in terms of meaning to the individual. We assume that places where users have meaningful stays are assigned roles, such as "home" or "work". A role differs from a place in that it has a unique interpretation for an individual. Most people have a place they call home, but (usually) few people call the same place home. A place may have a category that is the same for everyone (e.g., a grocery store), but a role that varies by individual (e.g., the place I go to work, the place I buy my food, or both). Similar to places and roles, transitions give meaning to travel segments. Common transitions that might be meaningful include going to work in the morning or taking the kids to swim lessons. Roles and transitions may be identified either by user-report or the system learning over time.

This context enables identifying room for improvement. For example, a common piece of advice to improve eating habits is to eat out at restaurants less. However, this advice does not apply to someone who never visits restaurants. A motivated individual provided with this advice will not be able to act on it, and could thereby negatively impact their selfefficacy to make changes. This makes them less likely to believe actually improve their eating habits overall.

Location can also improve understanding of causality. If a wearable device is collecting stress indicators, one may discover that even though she works long hours, she does not usually experience stress at work. However, the bowling alley after work does show elevated stress levels (competition play), while the bowling alley on Friday nights is again low stress (socializing).

\section{Behavioral Tendencies}

Once an individual's roles and transitions are identified, we can begin to identify patterns and trends that reflect behavioral tendencies. One approach is to identify consistent behaviors that location traces provide, such as always going to the same coffee shop in the morning, or always going to a different coffee shop. Once consistent behaviors are identified, they can be presented to the user as a means of interpretation and serve as a point for reflection. A system-provided interpretation (with supporting evidence) could provide an objective view on someone's lifelog. The user may or may not agree with the interpretation, but it could also become something that helps the user derive value from the lifelog.

One primary challenge around detecting behavioral tendencies reflected by patterns of locations is that routines take a while to learn. Different aspects of routine have varying periodicity. For example, a common routine might be to go to work every morning and return home every night. However, one night a week the individual may go to a yoga class before returning home. Other routines might have somewhat consistent periodicity, with some variation. One example is going to the grocery store: some people might go once a week fairly reliably, but the day or time may vary week to week.

Davidoff et al [11] were able to use calendaring information to predicts how likely a child in a family with two working parents will be forgotten to be picked up at an activity. Similar approaches could be applied to predict how likely a person would be to skip their workout today. This would enable a system to identify appropriate times for intervention or to present information.

Identifying behavioral tendencies over location, physical activity and food intake data can support the generation of insights to help people identify trends or behaviors they may want to change. For example, an insight that can identify that 
one always eats dessert at a particular restaurant. Or that a person always hits their step goal for the day when they take their kids to school, but never when they go directly to work. Data could also highlight how certain behaviors have impact on health and wellness indicators over a long period of time. While this position paper has focused primarily on health and well-being apart from chronic diseases, data collected over the long term can provide insight into the development of chronic diseases such as diabetes, heart disease or asthma.

In addition, the ability for the system to identify and model behavioral tendencies may allow for the system to identify drift over time, as well as identify factors that may have precipitated the begin of the drift. For example, it may be straightforward for the physical activity tracker to identify that you are exercising less, but the location stream can identify that you are spending more time at work.

\section{Aggregation and Cohort Identification}

The levels of interpretation described above each add unique value to the lifelog, however they also allow us to identify rich cohorts that can be used to answer questions of interest to the user. We imagine cohorts could be generated along a number of dimensions:

Demographic cohorts aggregate user data along any number of traditional demographic boundaries-age, sex, income, profession, etc.; augmenting this with the location dimensions discussed above adds geography, place category, travel duration, and transition data. Taken together with physiological data allows us to use these cohorts to answer questions such as: which cities burn the most calories? What state has the longest commutes? Which job categories are associated with the highest overall levels of stress? These can provide valuable insights into larger trends.

Potentially of more interest to the individual, behavioral cohorts can be organized around users with similar behavioral tendencies. These can combine aspects of demographic cohorts, can be much more user-tailored, e.g., "People who share my commuting pattern." These cohorts can be explored to help answer questions such as, "How do people who have my commute find time for physical activity?" or "How does my level of sleep and stress compare to others with a similar commute?" We believe these behavioral cohorts can provide a valuable capacity to discover new personal opportunities and new insights into one's own behavior.

Going beyond this first level of behavioral cohorts-e.g., "people like Alice"-we can also identify groups of individual who were like Alice, but have now changed their behavior in some dimension that Alice finds desirable. These cohorts can provide Alice with insight into how she might achieve that change herself. Clearly, as we further divide users into finer and finer cohorts, we will need an increasing number of participating users to find meaningful correlations. This points out the need for automated collection and incorporation of location data into the lifelog to acquire such a dataset.

\section{CONCLUSION}

Our goal is to identify and provide ways a lifelog can provide deeper value to users, particularly in the health and wellness domains. In this vein, we have presented the vision of a lifelog consisting of not only images, but location and data streams that reflect food consumption and physical activity. We believe that including an accurate, detailed location $\log$ in addition to these personal wellness streams can add value to the self-reflection process and support behavior change in these areas.

In this position paper, we described a phased approach to deriving meaning from location traces to support health and wellness monitoring and possibly behavior change. We offer this position as a contribution to the workshop, and hope to provide benefit to the workshop discussions.

\section{REFERENCES}

[1] A. R. Doherty, S. E. Hodges, A. C. King, A. F. Smeaton, E. Berry, C. J. A. Moulin, S. Lindley, P. Kelly, and C. Foster, "Wearable Cameras in Health: The State of the Art and Future Possibilities," American Journal of Preventive Medicine, vol. 44, no. 3, pp. 320-323, Mar. 2013.

[2] J. Rekimoto, T. Miyaki, and T. Ishizawa, "LifeTag: WiFi-based continuous location logging for life pattern analysis," Lecture Notes in Computer Science, vol. 4718, p. 35, 2007.

[3] D. Ashbrook and T. Starner, "Using GPS to learn significant locations and predict movement across multiple users," Personal and Ubiquitous Computing, vol. 7, no. 5, pp. 275-286, 2003.

[4] J. Hightower, S. Consolvo, A. LaMarca, I. Smith, and J. Hughes, "Learning and recognizing the places we go," in UbiComp 2005: Ubiquitous Computing, Springer, 2005, pp. 159-176.

[5] J. H. Kang, W. Welbourne, B. Stewart, and G. Borriello, "Extracting places from traces of locations," in Proceedings of the 2nd ACM international workshop on Wireless mobile applications and services on WLAN hotspots, 2004, pp. 110-118.

[6] L. Ferrari and M. Mamei, "Discovering daily routines from Google Latitude with topic models," in 2011 IEEE International Conference on Pervasive Computing and Communications Workshops (PERCOM Workshops), 2011, pp. 432-437.

[7] N. Eagle and A. Pentland, "Eigenbehaviors: identifying structure in routine," Behav Ecol Sociobiol, vol. 63, no. 7, pp. 1057-1066, May 2009.

[8] B. J. Fogg, Persuasive Technology: Using Computers to Change What We Think and Do (Interactive Technologies), 1st ed. San Francisco: Morgan Kaufman, 2002.

[9] F. Zhu, M. Bosch, I. Woo, S. Kim, C. J. Boushey, D. S. Ebert, and E. J. Delp, "The Use of Mobile Devices in Aiding Dietary Assessment and Evaluation," IEEE Journal of Selected Topics in Signal Processing, vol. 4, no. 4, pp. $756-766$, Aug. 2010.

[10] G. O’Loughlin, S. J. Cullen, A. McGoldrick, S. O'Connor, R. Blain, S. O'Malley, and G. D. Warrington, "Using a Wearable Camera to Increase the Accuracy of Dietary Analysis," American Journal of Preventive Medicine, vol. 44, no. 3, pp. 297-301, Mar. 2013.

[11] S. Davidoff, B. D. Ziebart, J. Zimmerman, and A. K. Dey, "Learning patterns of pick-ups and drop-offs to support busy family coordination," in Proceedings of the SIGCHI Conference on Human Factors in Computing Systems, New York, NY, USA, 2011, pp. 1175-1184. 